\title{
The Influence of Sustained Selective Attention on Stimulus Selectivity in Macaque Visual Area MT
}

\author{
Detlef Wegener, ${ }^{1}$ Winrich A. Freiwald, ${ }^{1,2,3}$ and Andreas K. Kreiter ${ }^{1}$ \\ ${ }^{1}$ Brain Research Institute, Center for Cognitive and Emotional Sciences, University of Bremen, D-28334 Bremen, Germany, ${ }^{2}$ Department of Brain and \\ Cognitive Sciences, Massachusetts Institute of Technology, Cambridge, Massachusetts 02139, and ${ }^{3}$ Department of Neurobiology, Harvard Medical School, \\ Boston, Massachusetts 02115
}

\begin{abstract}
Remarkable alterations of perception during long-lasting attentional processes have been described in several recent studies. Although these findings have gained much interest, almost nothing is known about the modulation of neuronal responses during sustained attention. Therefore, we investigated the effect of prolonged selective attention on neuronal feature selectivity. Awake macaque monkeys were trained to perform a motion-tracking task that required attending one of two simultaneously presented moving bars for up to 15 sec. Extracellular recordings were obtained from neurons in macaque motion-sensitive middle temporal visual area (MT/V5). Under conditions of attention, we found high and constant direction selectivity over time. This was expressed by a strong and persistent response contrast between presentations of preferred and nonpreferred stimuli in successive motion cycles. With attention directed to another moving bar, neuronal responses to the behaviorally irrelevant stimulus became continuously less specific for the direction of motion. In particular, increasingly higher firing rates for motion in null direction caused a strong reduction of direction selectivity, which further increased with enhanced proximity between target and distracter bar. A passive condition experiment revealed that this reduction occurred only when motion remained the behaviorally relevant feature but disappeared when attention was withdrawn from this feature domain. Thus, sustained attention seems to stabilize direction selectivity of neurons in area MT against a time and competitiondependent degradation, whereas nonattended objects suffer from a reduced neuronal representation.
\end{abstract}

Key words: macaque monkey; vision; MT; attention; direction selectivity; neurophysiology

\section{Introduction}

A number of psychophysical studies in the field of inattentional blindness demonstrated that subjects often fail to notice clearly visible objects when they are engaged in a demanding attention task (Neisser, 1967). In a recent experiment, Simons and Chabris (1999) showed that subjects may even fail to report the presence of a person disguised as a gorilla who is walking and jumping among basketball players if they are required to count the passes between players of one of two teams. In a more formalized account, Most et al. (2001) found that a visual stimulus that differs in both color and form from all other objects on the display may not reach perceptual awareness when it is not attended, even when it moves through the center of gaze. These results are especially surprising because, under neutral viewing conditions, one would expect salient objects to be immediately perceived. Therefore, it has been suggested that neuronal processing of stimuli can be strongly reduced when these are outside the focus of

Received April 19, 2004; revised May 21, 2004; accepted May 22, 2004.

This work was supported by SFB 517 of Deutsche Forschungsgemeinschaft and by grants from Hans-BöcklerStiftung (D.W.), Volkswagenstiftung (W.A.F.), and Hanse Institute for Advanced Study (W.A.F.). We are grateful to Anja Besuch, Barbara Klein, Katrin Thoss, and Sigrun Wicker for technical assistance and animal care and to Nicole Strüber for training assistance. Sunita Mandon and Michael Koch provided helpful comments on this manuscript.

Correspondence should be addressed to Dr. DetlefWegener, Brain Research Institute, University of Bremen, P.O. Box 3304 40, D-28334 Bremen, Germany. E-mail: wegener@brain.uni-bremen.de. DOI:10.1523/JNEUROSCI.1459-04.2004

Copyright $\odot 2004$ Society for Neuroscience $\quad$ 0270-6474/04/246106-09\$15.00/0 attention, preventing them from being consciously perceived (Mack and Rock, 1998; Simons and Chabris, 1999).

An important property of many studies in the field of inattentional blindness is that they require subjects to do a demanding attention task for prolonged periods of time before the test stimulus is presented (Neisser and Becklen, 1975; Becklen and Cervone, 1983; Stoffregen et al., 1993; Simons and Chabris, 1999; Most et al., 2001). In everyday life, such long-lasting attentional processes play an important role. Whenever we read a difficult text or do fine mechanical work, like the watchmaker who is repairing the clockwork, we have to "concentrate," and this is often accompanied by the subjective feeling of diminished awareness for everything not belonging to our current behavioral goal. In other words, long-lasting allocation of attention on a certain task can be paralleled by reduced perception of other stimuli that are consistently outside the focus of attention. This raises the question of how neuronal processing is modified by sustained attention.

To study the influence of sustained attention on neuronal processing, we used a motion-tracking task previously introduced by Treue and Maunsell (1996). We favored this task because, first, it is known to cause attentional modulation of neuronal activity in macaque middle temporal visual area (MT) and second, it uses moving stimuli, which are not supposed to be the subject of a fading process as a result of perceptual adaptation (May et al., 2003). The motion-tracking task is therefore well 


\section{Distant-Bar Experiment}

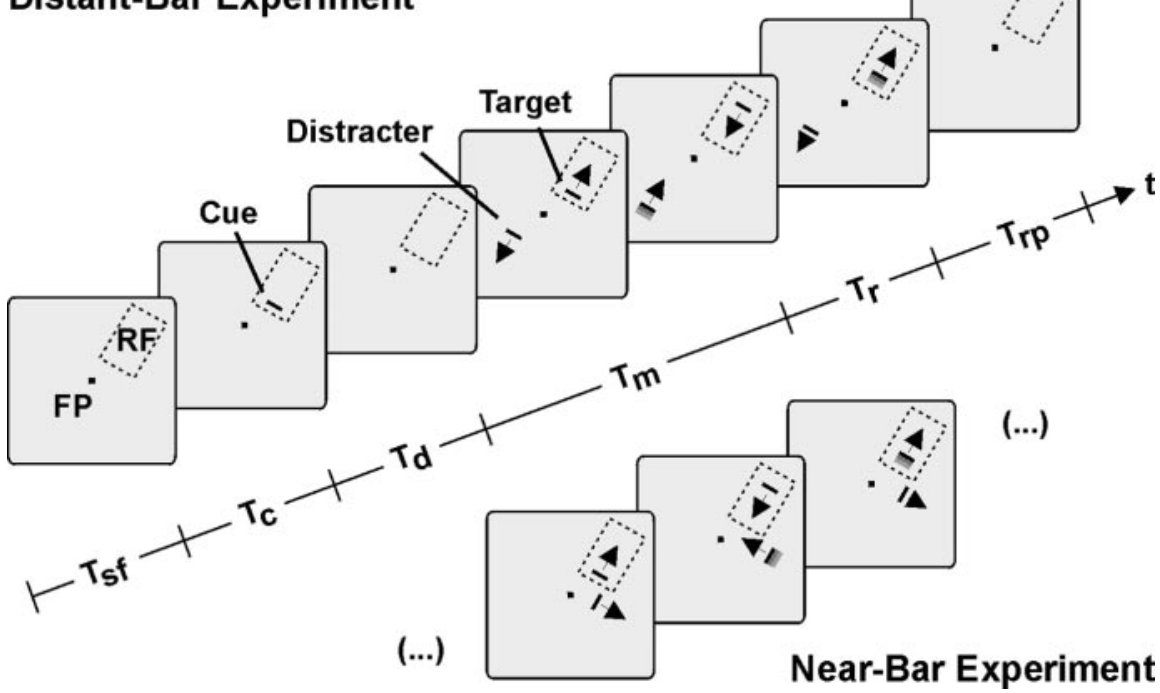

Figure 1. Sequence and spatial arrangement of the stimuli for distant-bar and near-bar experiments. Both experiments followed the same temporal sequence, but the two bar stimuli were arranged differently in terms of spatial separation and relative motion directions: one bar was always placed inside the RF of the recorded neuron and moved back and forth along a trajectory matched to the preferred direction. The second bar was placed with identical eccentricity in the opposite hemifield and moved in counter-phase in the distant-bar experiment. In the near-bar experiment, the second bar was positioned in the vicinity of the RF and moved along an orthogonal path. Each trial started with the appearance of a fixation point (FP). If the monkey responded by starting fixation and pressing a lever within period $T_{\text {sf }}$, the trial continued with a 500 msec presentation of a spatial cue $\left(T_{c}\right)$, indicating the location of the target. After a delay period $\left(T_{\mathrm{d}}\right)$, both bars appeared and started immediately to move for up to 15 $\sec \left(T_{\mathrm{m}}\right)$. At the end of $T_{\mathrm{m}}$, the target bar increased its speed, and a response period $\left(T_{\mathrm{r}}\right)$ of $500 \mathrm{msec}$ (monkey $\mathrm{K}$ ) or $700 \mathrm{msec}$ (monkey E) followed, during which the animal had to release the lever. Successive trials were separated by a resting period $\left(T_{r p}\right)$ of 2 or $3 \mathrm{sec}$. In approximately half of the trials, speed-up of the target was preceded by speed-up of the distracter bar, which had to be ignored. Acceleration is indicated by shading of the bars.

suited for long-lasting presentation. The results of our experiments show, that the ability of neurons in area MT to represent the direction of motion is strongly reduced when attention is withdrawn from the stimulus. In contrast, for attended objects, neuronal selectivity is high and remains constant even when stimulation lasted for prolonged periods of time. Preliminary results of this work have been published previously in abstract form (Wegener et al., 2003).

\section{Materials and Methods}

The effect of attention on direction selectivity of neurons in area MT was investigated by recording from two adult male monkeys (Macaca mulatta). During training and recording sessions, the animals sat in a primate chair with the eyes $80 \mathrm{~cm}$ in front of a 22 inch monitor (Elsa Ecomo750; Elsa AG, Aachen, Germany) and the head restrained. Stimuli were generated on a PentiumIII computer with a Nvidia Geforce2 graphics card. Eye position was monitored by a modified eye movement monitor (E5; Remmel Labs, Ashland, MA) using the double magnetic induction method (Allik et al., 1981; Bour et al., 1984). The resolution of this method was $0.3^{\circ}$ for eye movements along the vertical axis and $0.25^{\circ}$ along the horizontal axis.

Behavioral paradigm and visual stimulation. The animals were trained to perform a motion-tracking task adapted from Treue and Maunsell (1996), as illustrated in Figure 1. Briefly, animals were required to attend to one of two moving bar stimuli and to detect a slight acceleration of the attended bar while fixating a small spot at the center of the screen. Each trial started with the appearance of a red $0.15 \times 0.15^{\circ}$ fixation spot, requiring the animal to start fixation and to press a lever within a time window of $3 \sec \left(T_{\mathrm{sf}}\right)$. When the animal kept fixation for half a second, $T_{\mathrm{sf}}$ terminated and a stationary bar was presented for $500 \mathrm{msec}\left(T_{c}\right)$ at one of two possible positions. The bar served as a cue for the position of the target stimulus in this trial. After another 500 or $1000 \mathrm{msec}$ delay $\left(T_{\mathrm{d}}\right)$, the target bar appeared in the cued position and a distracter bar at the other uncued position. Both bars moved back and forth along a trajectory of $1.4-2.1^{\circ}$ visual angle with identical velocity chosen between 1.4 and $1.8 \%$ sec. After a random time interval chosen independently for each bar between 0.5 and $15 \mathrm{sec}\left(T_{\mathrm{m}}\right)$, velocity increased by $40-60 \%$. The animals were required to respond to an increase of the velocity of the target bar by releasing the lever within a response interval $\left(T_{\mathrm{r}}\right)$ of $500 \mathrm{msec}$ (monkey K) or $700 \mathrm{msec}$ (monkey E) but to ignore an acceleration of the distracter bar, which occurred in part of the trials before acceleration of the target. Correct performance was rewarded with fruit juice. Release of the lever too early or too late with respect to the response interval or deviation of eye position from the fixation point by $>0.75^{\circ}$ in horizontal or vertical direction caused the immediate termination of the trial accompanied by acoustic feedback signals. Successive trials were separated by a resting period $\left(T_{\mathrm{rp}}\right)$ of $2-3 \mathrm{sec}$. On every trial, one of the bars was located over the receptive field $(\mathrm{RF})$ of the recorded cell, the other was placed either with identical eccentricity in the opposite hemifield [distant-bar experiment (DB)] or close to but still outside the RF [nearbar experiment (NB)]. In the DB experiment, motion of the bar outside the RF was always in the opposite direction compared with the direction of the bar inside the RF. In the NB experiment, it moved in orthogonal direction.

In addition to the $\mathrm{DB}$ and $\mathrm{NB}$ experiment, we recorded neuronal responses under another behavioral condition, termed "passive condition" for the rest of this study. Here, animals were required to keep fixation and to detect a small dimming of a white fixation spot at the end of the trial. The neurons under investigation were stimulated with a behaviorally irrelevant bar moving back and forth over the RF with the same parameters as in the experiments described above but with a fixed interval of stimulus presentation allowing to record responses during three complete cycles of forward and backward motion.

Surgery. After completion of the initial fixation training, the animals were implanted with a head post and recording chambers of $20 \mathrm{~mm}$ diameter. For surgery, initial anesthesia was induced by intramuscular injection of ketamine $(10 \mathrm{mg} / \mathrm{kg})$ supplemented with xylacine $(0.04 \mathrm{mg} /$ $\mathrm{kg}$ ) and was continued with $0.5-3 \%$ isofluorane in oxygen/nitrous oxide (30/70) after tracheal intubation. Recording chambers were oriented in the parasagittal plane with a $30^{\circ}$ angle to the horizontal plane. The center was placed $15 \mathrm{~mm}$ lateral to the midline of the skull and the posterior border near the occipital ridge. Bone screws, head post, and recording chamber were fixed and interconnected by bone cement (Palacos; Merck, Darmstadt, Germany) and dental acrylic cement (Paladur; Heraeus, Hanau, Germany). During a second surgery, a $17 \mathrm{~mm}$ diameter gold plated ring was implanted between sclera and conjunctiva of one of the eyes (Fuchs and Robinson, 1966; Judge et al., 1980). Surgical procedures were conducted under aseptic conditions. Postoperative treatment included prophylactic administration of antibiotics and a recovery period of 6 weeks. All surgical procedures and experimental techniques were performed in accordance with the National Institutes of Health Guide for Care and Use of Laboratory Animals and the regulations for the welfare of experimental animals issued by the Federal Government of Germany and had been approved by the local authorities.

Recording technique. Single-unit and multiunit recordings were obtained from area MT in three hemispheres using varnish-coated microelectrodes with an impedance of $0.8-1.7 \mathrm{M} \Omega$ at $1 \mathrm{kHz}$. The signal of the electrode was amplified by a factor of $10,000-50,000$ and bandpass filtered from 0.7 to $5 \mathrm{kHz}$. The waveform of each spike that crossed threshold was recorded together with continuous traces of the eye position 
signals. Isolation of single units was improved by off-line spike-sorting techniques on the basis of waveform parameters such as principal components, amplitude, or slope. In this way, $>50 \%$ of all units analyzed in this study were characterized as single units. Of the remaining recordings, most were dominated by a single unit, and for a minority of recordings, we were not able to further sort the recorded waveforms. Area MT was identified by its position in the superior temporal sulcus, by the high proportion of direction selective neurons, the size/eccentricity ratio of the RFs, the typical sequence of RF locations along the electrode tracks, and its position relative to adjoining areas (Maunsell and Van Essen, 1983; Desimone and Ungerleider, 1986). The extent of the RFs, approximated by rectangles, and their preferred direction of motion were determined by manual mapping with light bars.

Data analysis. To assess attention-dependent changes of direction selectivity over time, we computed the direction index (DI) of neuronal responses during successive motion cycles separately for different conditions of attention. A motion cycle consisted of motion in preferred followed by motion in anti-preferred (null) direction. The DI was estimated from the response rates to preferred $\left(R_{\mathrm{P}}\right)$ and null motion $\left(R_{0}\right)$ as follows:

$$
D I=\left(R_{\mathrm{P}}-R_{\mathrm{o}}\right) /\left(R_{\mathrm{P}}+R_{\mathrm{o}}\right) .
$$

The index varies between 0 , if responses to both directions of motion do not differ, and 1 , if responses occur in preferred direction only. Values between 0 and -1 may result if responses are modulated in a way that preferred motion elicits less spiking activity than motion in nonpreferred direction. Note that we did not subtract spontaneous activity because, for the nonattended condition, this procedure often generated values not confined to the mathematically defined domain of the function delivering the DI. Such values resulted from cells with small $R_{\mathrm{P}}$ and negative $R_{0}$, thus leading to a denominator close to or identical to zero and hence a DI that is not meaningful.

Response rates were estimated within separate time windows for forward and backward motion. The average rate within these windows was computed from peristimulus time histograms (PSTHs) with $1 \mathrm{msec}$ resolution on the basis of responses that occurred before any bar accelerated in correctly performed trials. To ensure correct assignment of spikes to phases of motion in preferred and null direction, two adjustment operations were applied to the windows. First, to account for the response delay of each neuron, analysis windows were shifted by the minimal onset delay. Second, analysis windows were further restricted, because the length of both on and off response delays was variable. We computed the difference between maximal and minimal delay and than added this value to each analysis windows beginning and subtracted it from the end. This procedure prevented false spike assignment and guaranteed identical RF coverage during preferred and null motion analysis periods. The same analysis windows were then used for each motion cycle and condition of attention.

Computation of response delays was based on smoothed PSTHs, which had been obtained by a convolution of the original PSTH with a Gaussian kernel ( $\sigma=10 \mathrm{msec}$, except for 10 of 122 cases in the passive condition for which $\sigma=20 \mathrm{msec}$ ). Response onset was defined as the time between stimulus onset and the first bin exceeding the $p=0.01$ level for surpassing spontaneous activity. This level was found by computing the Poisson distribution that described the values in the 150 bins immediately before stimulus onset. Offset delay was computed analogously after time inversion of the PSTH. The method yielded values close to those obtained with the method of Maunsell and Gibson (1992) but turned out to be more robust for the present data set.

To investigate differences between effects of attention on responses to motion in preferred and in null direction and their change over time, an attention index (AI) was computed separately for each motion trajectory:

$$
A I=\left(R_{\mathrm{A}}-R_{\mathrm{NA}}\right) /\left(R_{\mathrm{A}}+R_{\mathrm{NA}}\right) .
$$

The response rates in the attended $\left(R_{\mathrm{A}}\right)$ and nonattended $\left(R_{\mathrm{NA}}\right)$ conditions were estimated for consecutive motion trajectories as described above for estimation of the DI. The index is equal to one if a cell responded to the attended stimulus only, and zero if responses to attended and nonattended objects did not differ. It reached negative values if responses for the nonattended stimuli exceeded those of attended stimuli.

In addition to the calculation of indices, we also calculated a ratio for both the analysis of direction selectivity (DR) and of attentional modulation $(\mathrm{AR})$ :

$$
\begin{aligned}
& \mathrm{DR}=R_{\mathrm{P}} / R_{\mathrm{O}} . \\
& \mathrm{AR}=R_{\mathrm{A}} / R_{\mathrm{NA}} .
\end{aligned}
$$

Statistical significance of changes in directional selectivity was tested in two ways. For population data, we performed the Wilcoxon rank sum test for paired differences on the DI computed for different behavioral conditions in each motion cycle. To assess whether an individual neuron showed a significant attention-dependent change of its DI over time, we used the following procedure: for each neuron, we computed a stability ratio for directional tuning by dividing the DI of the nth motion cycle by the DI of the first motion cycle. This ratio is close to one if directional tuning remains essentially unaltered. From the population of neurons recorded in the fixation condition, for which the mean DI was found to be stable over time, we obtained a set of ratios with Gaussian distribution for the second and third motion cycle. Based on these distributions, it was determined whether the stability ratio of a given neuron deviated significantly $(p<0.05)$ from one, indicating a significant change of its direction selectivity.

\section{Results}

\section{Behavioral performance}

The behavioral paradigm required the animals to pay sustained attention to an extrafoveally presented bar for up to $15 \mathrm{sec}$ without moving the eyes. Despite the great behavioral demands associated with long-lasting attentional tracking, both animals showed satisfying behavioral performance. In the DB experiment, the average performance of monkeys $\mathrm{K}$ and $\mathrm{E}$ was $\sim 84$ and $75 \%$, respectively, ignoring trials that were aborted because of eye movements (monkey K, 25\%; monkey E, 33\%). The majority of errors of monkey $\mathrm{K}$ resulted from trials in which the animal failed to indicate target acceleration within the response period (11\%). Responses before target acceleration occurred only rarely. For monkey E, most errors resulted from trials where the animal released the lever before target acceleration occurred (18\%). Within the NB experiment conducted with animal $K$, the average performance was $75 \%$, and the main error source was again a failure to release the lever during the response period (21\%). In the passive condition, average performance was $88 \%$ for monkey $\mathrm{K}$ and $85 \%$ for monkey $\mathrm{E}$. With respect to the directedness of covert attention, it is important to notice the reduced number of eye errors, which were $9 \%$ in monkey $\mathrm{K}$ and $12 \%$ in monkey $\mathrm{E}$. Most of these errors occurred in only a few recordings in which the animals were already tired. More than $50 \%$ of all recordings in this condition were done with zero eye errors.

\section{Attention-dependent modulation of direction selectivity}

The selectivity of MT neurons for the direction of motion was strongly influenced by sustained visual attention. When presented with two competing bars, we found high and constant direction selectivity when the animals attended the bar over the RF. In contrast, stimulation of the RF with nonattended bars yielded lower neuronal selectivity that decreased progressively over time the longer the animal attended the bar outside the RF. This effect is illustrated in Figure $2 A$, which shows the response patterns of a single neuron recorded in the DB experiment. The neuron responded to the presentation of successive motion cycles, each consisting of motion in preferred direction followed by motion in null direction. The contrast between responses to preferred and null motion was quantified by the DI, which served as a measure of the selectivity of the cell. With attention directed to 
A

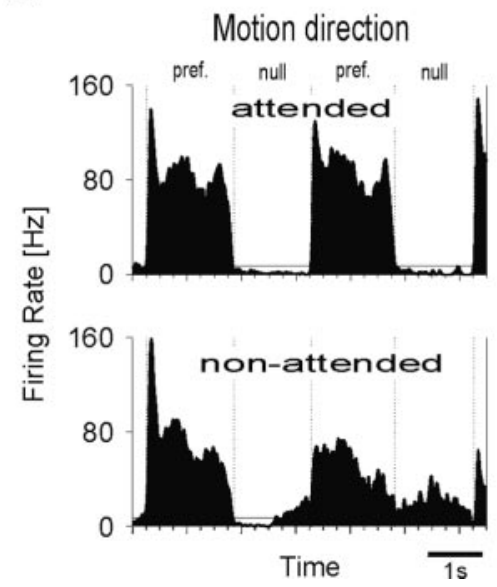

B

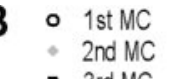

- 3rd MC

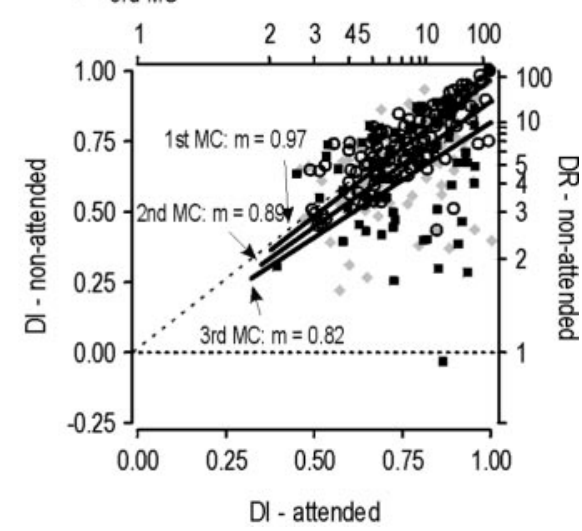

Figure 2. Distant-bar experiment. A, Example for attention-dependent modulation of responses to backward and forward motion in a single MT neuron. Dotted vertical lines represent the beginning and end of a response for motion in preferred (pref.) or anti-preferred (null) direction. Throughout this study, each pair of subsequent preferred and null motion is called a motion cycle. The top PSTH shows that with attention directed to the RF, stimulation with preferred motion elicited a high firing rate, which stayed so during both cycles. Stimulation with null motion yielded responses constantly below spontaneous activity. The bottom PSTH shows the response of the same cell with attention directed away from the RF. Responses for preferred motion were reduced compared with the attended condition, but responses to null motion showed an increase, which rose over time. This attention-dependent modulation of firing rates led to a strong reduction of the response contrast for motion in preferred and anti-preferred direction and, thus, to a decrease of direction selectivity. See Results for additional details. $B$, Attentional modulation of direction selectivity for all neurons. The plot shows DI and DR of the nonattended condition versus the attended condition. The dotted line is the bisector; the dashed horizontal line marks the zero value for the DI of nonattended motion. Straight lines represent the mean ratio for the first, second, and third motion cycles $(M C s)$, and arrows relate the value of each slope $(m)$ to the appropriate line.

the bar inside the RF of the neuron, the firing rate of this cell changed repeatedly between a high discharge rate of $\sim 60 \mathrm{~Hz}$ for the preferred direction of motion and a very low rate of $\sim 1 \mathrm{~Hz}$ for the opposite direction of motion. This response pattern did not change between the two successive motion cycles, resulting in almost identical DIs of 0.94 and 0.95 , indicating high and sustained direction selectivity under conditions of attention. For the nonattended condition, with attention directed to the bar in the opposite hemifield, the response pattern of the cell is altered in two ways, both of which gain strength over time. First, the response magnitude to the preferred motion direction dropped to $50 \mathrm{~Hz}$ in the first and further to $35 \mathrm{~Hz}$ in the second motion cycle. Second, responses to motion in null direction followed the opposite trend and increased to 5 and $14 \mathrm{~Hz}$, respectively. This surprising result shows that for nonpreferred stimuli, responses to nonattended stimuli might become stronger than those to attended stimuli. As a consequence, without attention, the contrast between responses to preferred and to null motion reduces progressively over time. This is reflected in directional indices dropping to 0.83 and 0.44 for the first and second motion cycle, respectively, corresponding to a reduction of 12 and 54\% compared with the attended condition. Thus, the stimulus selectivity of the neuron reduces strongly with attention permanently withdrawn from the RF.

For the DB experiment, we obtained quantitative data of responses during the first, second, and third motion cycle from 100, 95 , and 73 neurons, respectively. Figure $2 B$ shows the relation between the DIs of individual units in the attended and the nonattended condition. For individual motion cycles, a straight line was fitted, constrained to pass zero, which represents the mean of the ratio between the DIs of individual units. The slope of each line is $<1.0$, and the smallest value was obtained for the third motion cycle. Across attentional conditions, this illustrates weaker direction selectivity for nonattended stimuli, becoming increasingly worse from the first to the third motion cycle. Analysis of the population data within each condition revealed that over the course of three consecutive motion cycles, mean direction selectivity stayed high and essentially constant if the stimulus was attended $\left(\mathrm{DI}_{\mathrm{At}}, 0.76,0.75,0.76\right)$. In contrast, with attention directed away from the RF, the mean DI decreased by $8.1 \%$ and $14.9 \%$ for the second and third motion cycles compared with the first $\left(\mathrm{DI}_{\mathrm{NA}}\right.$, $0.74,0.68,0.63)$. With respect to the attended condition, this was a highly significant reduction of the mean DI by $9.3 \%$ $(p<0.005$; Wilcoxon rank sum test; $n=$ $95)$ and $17.1 \%(p<0.0001$; Wilcoxon rank sum test; $n=73$ ) in the last two motion cycles.

To assess the number of individual cells showing a significant change in direction selectivity over time, we tested the respective DI values of the second and third motion cycles against the first one (see Materials and Methods). Figure 3 summarizes the results of this analysis. The scatter plots illustrate the time-dependent relationships of the DIs separately for the attended and nonattended conditions. Open circles indicate neurons with unchanged direction selectivity over time. Filled circles indicate neurons that underwent significant changes of the DI, either increasing over time (gray) or decreasing (black). With attention directed to the RF, the vast majority of cells showed unchanged direction selectivity. This was found for $93 \%$ of the neurons when comparing first and second motion cycles and for $84 \%$ when comparing first and third cycles. Of the few remaining cells, some showed a decrease of selectivity, and others became somewhat more selective.

In contrast, constancy of direction selectivity was lost for a large fraction of cells in the nonattended condition. In the second motion cycle, $28 \%$ had a significantly reduced DI compared with the first, and in the third cycle, this proportion further increased to $>50 \%$. Thus, with persistent allocation of attention elsewhere, increasingly more cells showed a reduction of direction selectivity, indicating the prevalence of this effect within the population of MT neurons.

A reduction of processing quality for unattended stimuli is often attributed to competition between multiple stimuli for limited neuronal resources (Desimone and Duncan, 1995). If the reduced direction selectivity observed for nonattended stimuli depends on the competition between the two bars, then an even stronger reduction of the DI is expected when competition is enhanced. We aimed to test this possibility in an additional experiment by decreasing the spatial distance between the two bars (NB experiment), a modification of the stimulus constellation, which is generally assumed to enhance interstimulus competition. The bar outside the RF, originally located in the opposite hemifield, now described a trajectory close to the RF. To minimize modulatory effects of the neuronal responses from the surround of the RF (Allman et al., 1985), we chose a direction orthogonal to the cells preferred direction of motion. Figure $4 \mathrm{~A}$ gives an example of the response properties of a single neuron under 
these conditions. Again, with attention directed to the RF, the cell showed high response rates of $\sim 100 \mathrm{~Hz}$ for preferred motion and weak responses below spontaneous activity if stimulated with null motion. This gave rise to high and essentially constant DI values of 0.87 and 0.84 for the first and second motion cycles, respectively. In the nonattended condition, the response rate dropped to 36 and $28 \mathrm{~Hz}$, respectively, when the stimulus moved in the preferred direction of the cell. However, responses to null motion increased strongly, reaching approximately the same firing rates that were elicited by the preferred stimulus. Consequently, the DI values dropped to 0.05 and -0.06 for the first and second motion cycles, indicating that the cell essentially lost its ability to differentiate between preferred and null motion.

Analysis of the population data confirmed a general increase of the effects already observed in the DB experiment. We obtained quantitative data from 37 neurons for the first and second motion cycles and from 33 neurons for the third motion cycle. The relationship of the DIs across both conditions of attention is shown in Figure $4 B$. First, fitting a straight line to the points of each motion cycle again indicates weaker direction selectivity for stimuli outside the focus of attention. Second, comparison with the DB experiment shows that attenuation of direction selectivity is much stronger than in the DB experiment. Third, reduction of the DI raised strongly over time as indicated by the progressively decreasing slope of the fits to successive motion cycles. Consistently, separate analysis of the DI within each attention condition revealed a qualitatively different development of direction selectivity (Fig. 5). As in the previous experiment, we found that the average DI for the attended condition stayed high and essentially constant during the three consecutive motion cycles $\left(\mathrm{DI}_{\mathrm{At}}, 0.77,0.76,0.75\right)$. Accordingly, in $>90 \%$ of the cells, direction selectivity did not change significantly, even when we compared the first with the third motion cycle. In contrast, up to $88 \%$ of the neurons reduced their stimulus selectivity over time if attention was withdrawn from their stimulus. For the entire population, this went along with a decrease of the average DI by $30 \%$, and in comparison to the attended condition, we found the DI reduced by 13,25 , and $37 \%$ during the three consecutive motion cycles $\left(\mathrm{DI}_{\mathrm{NA}}, 0.67,0.57,0.47\right)$. The progressive change of mean direction selectivity along the time axis is highly significant ( $p<0.0001 ; n=33$; Wilcoxon rank sum test), and it is more than twice as strong as in the DB experiment, indicating that stimulus selectivity indeed suffers from increased spatial competition. attended
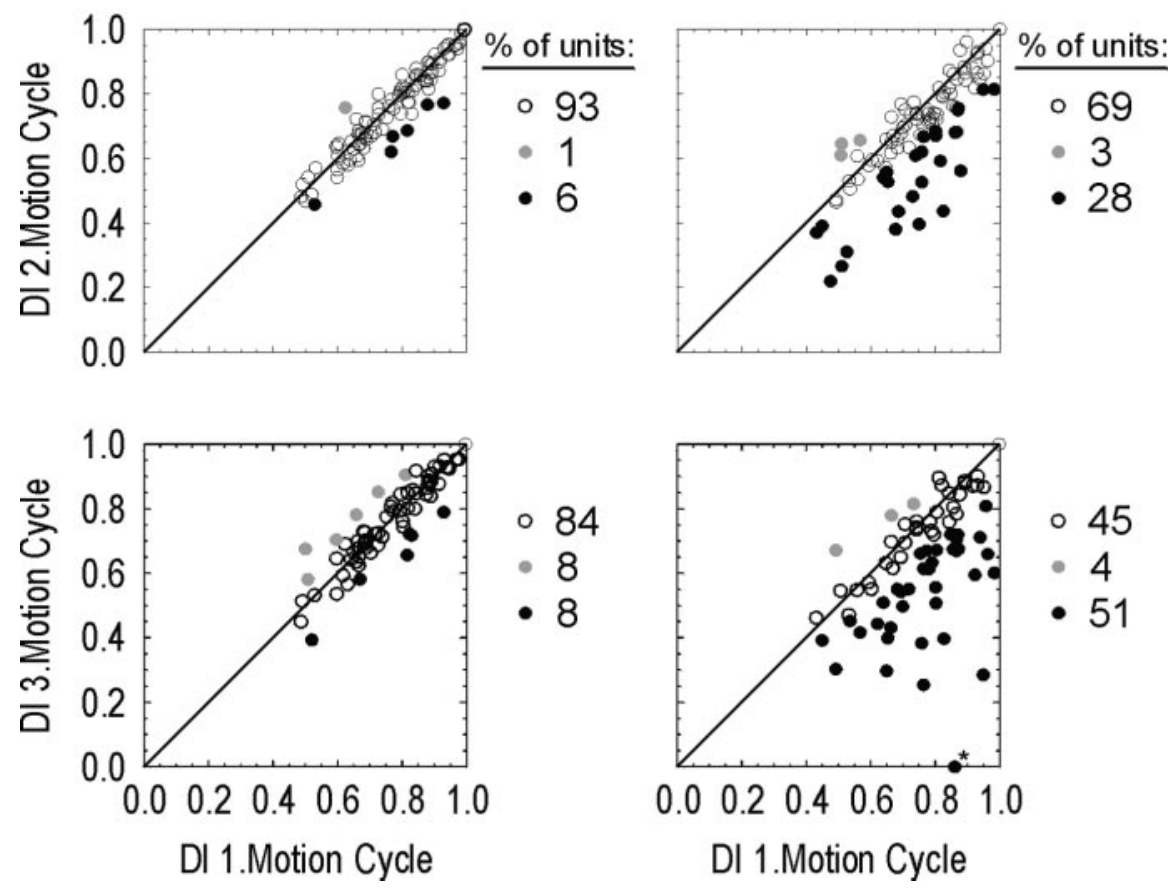

Figure 3. Change of direction selectivity over motion cycles in the DB experiment. The plots show the DI of all units for the second (top row) and third (bottom row) motion cycles against the first one with attention either directed to the RF (left column) or away from it (right column). Open circles, Selectivity not significantly changed over time; filled gray circles, selectivity significantly increased; filled black circles, selectivity significantly decreased. Numbers to the right of the scatter plots indicate the proportion of these cases within the population. The plots show that the majority of neurons recorded in the attended condition have constant direction selectivity over time. For the nonattended condition, $28 \%$ of neurons in the second motion cycle and $51 \%$ during the third one showed significantly reduced direction selectivity. The asterisk indicates a neuron for which the corresponding data point was placed on the null axis, although the neuron had a negative DI value $(-0.03)$ in the third motion cycle.
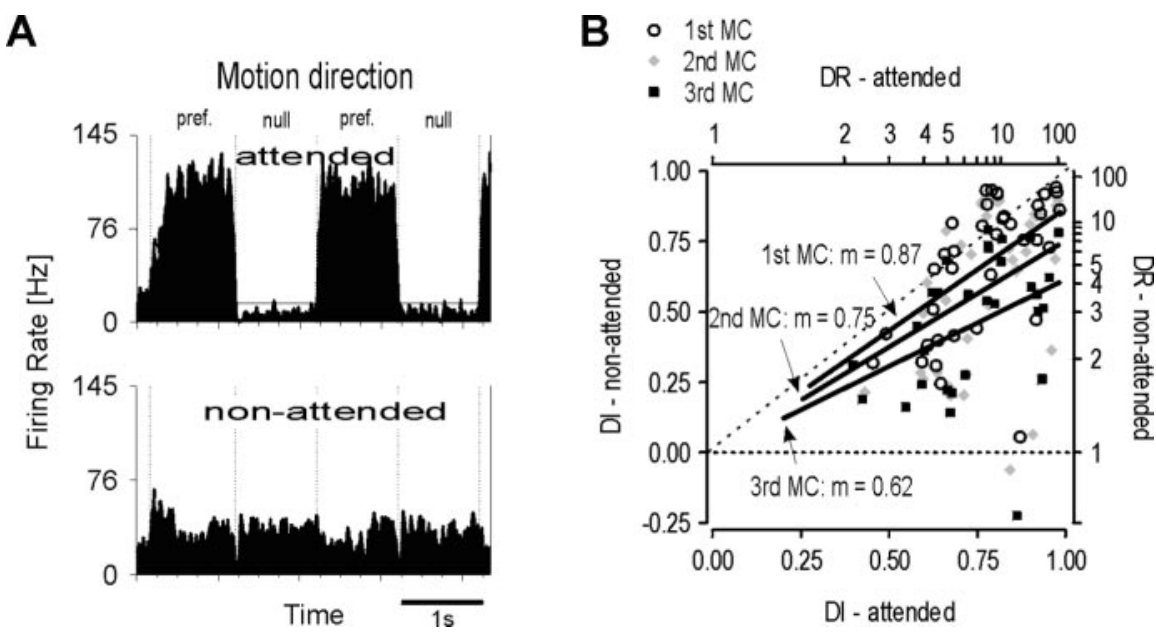

Figure 4. Near-bar experiment. Conventions are the same as in Figure 2. $A$, For the attended condition (top histogram), this single unit showed strong direction selective responses, but with attention directed away from the RF (bottom histogram), direction selectivity disappeared. Note that this is caused not only by a decrease of responses to preferred motion but by a multifold increase of responses to nonpreferred motion. See Results for details. B, Attention-dependent modulation of direction selectivity in the population of MT neurons during the first three motion cycles (MCs). Straight lines fitted to the data points of each MC indicate strongly decreasing direction selectivity when attention is withdrawn from the RF with the highest reduction of $\sim 40 \%$ during the third cycle.
Comparison with passive stimulation

The strong modulatory effect of attention on the direction selectivity of the neuron could either result from enhanced selectivity in the attended condition or, alternatively, from a degradation of selectivity when attention is located elsewhere. To test for neuro- 
attended
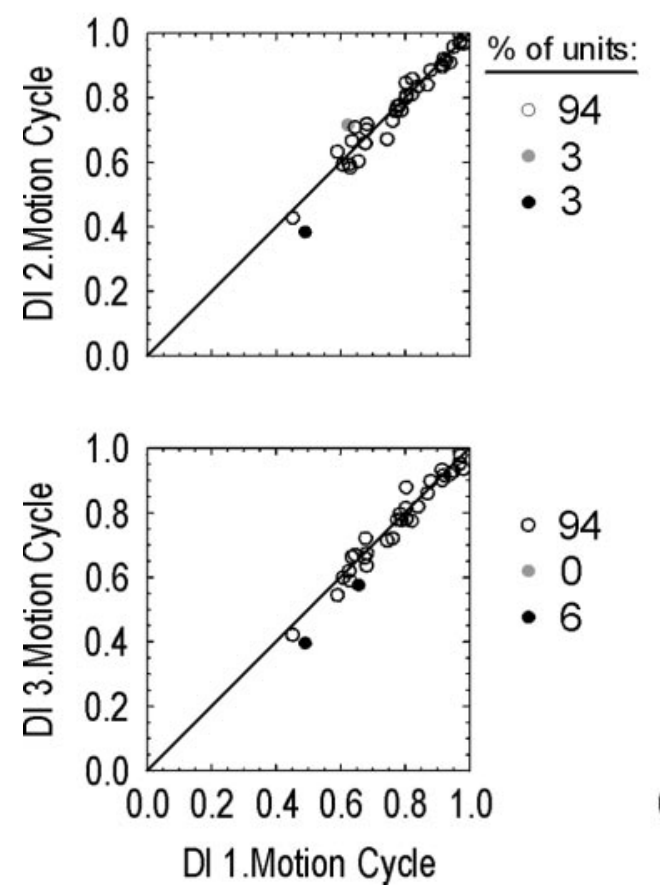

non-attended
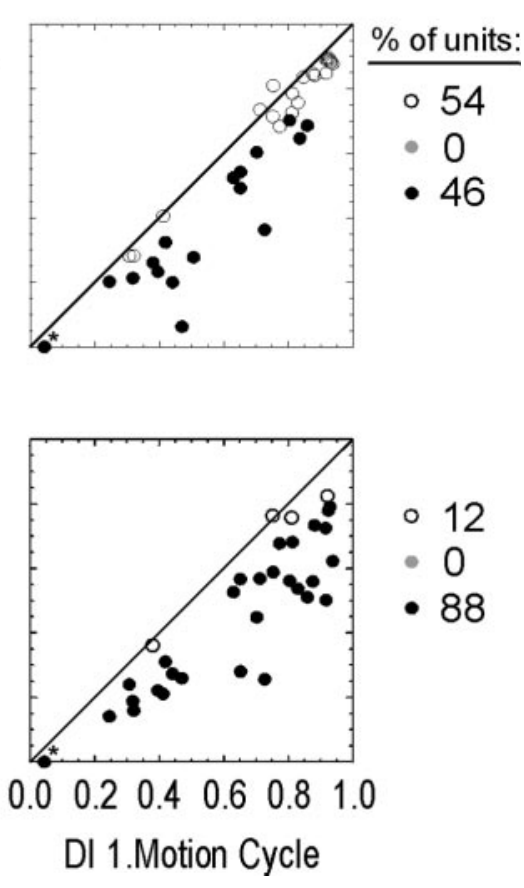

Figure 5. Change of direction selectivity over motion cycles in the NB experiment. Conventions are the same as in Figure 3. Note that for the attended condition, direction selectivity is preserved over time, whereas for the nonattended condition, $46 \%$ of neurons in the second motion cycle and $88 \%$ of the neurons in the third cycle showed a significant decline of the DI compared with the first MC. Asterisks indicate a neuron with negative DI values during second and third MC in the nonattended condition ( -0.06 ; $-0.23)$ for which the corresponding data point had been placed on the null axis for simplicity.

nal direction selectivity without motion processing supported by attention, we conducted a passive condition experiment that was characterized by, first, the absence of a competing motion stimulus and, second, the behavioral insignificance of the moving object. Thus, we expected undisturbed and purely sensory-driven processing, which provides a reference for the direction of attentional modulation. The passive condition experiment consisted of a fixation task, whereas preferred and null motion of a behaviorally irrelevant bar was presented to the RF of the neuron. A typical example for this experiment is illustrated in Figure 6, which shows the response of a neuron for two consecutive motion cycles. The firing rate for motion in preferred direction was high and showed no distinct change during the course of stimulation. Likewise, the responses for motion in the nonpreferred direction stayed low during both cycles, also indicating that under passive viewing conditions, no time-dependent modulation of the responses occurred. Hence, a high and constant DI characterized the response of the cell, even though no benefit can be gained from attention-dependent mechanisms in the passive condition.

Quantitative data for the first, second, and third motion cycles of the passive condition were obtained from 122, 117, and 93 neurons, respectively. The mean DIs for the three motion cycles $(0.78,0.79,0.78)$ confirmed that direction selectivity stays high and constant for moving stimuli, which are no targets of attention. The results of this and the two previous experiments are summarized and compared in Figure 7. We found a clear correspondence between the passive condition and the attended conditions of both the DB and NB experiments with respect to the flat time course and the high level of direction selectivity. In these conditions, there is either no competition or it is resolved by selective attention in favor of the stimulus inside the RF of the neuron. In contrast, neurons representing a nonattended stimulus show a progressive decrease of direction selectivity during sustained attention to the motion of another stimulus. Only in the first motion cycle of the distant bar configuration (i.e., low spatial competition) did we find neuronal selectivity to be comparable with that of the attended condition. For ongoing periods of time as well as increased spatial competition, direction selectivity was strongly reduced.

\section{Separate analysis of preferred and null motion}

An increase in directional selectivity may be caused by enhanced firing rates during motion in preferred direction or by decreased firing rates for motion in nonpreferred direction. To assess whether the attention-dependent change of the DI was primarily attributable to a modulation of responses to one of both directions of motion, or whether responses to both of them were equally modulated, we calculated the AI for each direction of motion. Analysis of the AI revealed that DI reduction was caused by the modulation of firing rates to both directions of motion, but its striking time course was primarily attributable to the increase of firing rates to nonpreferred motion (Fig. 8). In the DB experiment, the AI for motion in the preferred direction showed a moderate, although significant, increase over time $\left(\mathrm{AI}_{1-3}, 0.09,0.10,0.11 ; p<0.05\right.$ Wilcoxon rank sum test; $n=90)$. In the LC experiment, the AI increased from the first $(0.26)$ to the second motion cycle (0.33) but was essentially unchanged from the second to third cycle (0.32). However, this increase did not reach statistical significance. In contrast, for motion in nonpreferred direction, we found continuously decreasing values of the AI. In the DB experiment, the AI dropped from a positive value for the first phase of motion in nonpreferred direction to negative values for the second and third phase $(0.05$, $-0.01,-0.12)$, indicating stronger responses to null motion in the nonattended than the attended condition. The results from the NB experiment followed a similar pattern but reached an even more negative AI value during the third motion cycle $(0.04$, $-0.03,-0.18)$. In both experiments, the drop of the AI from first to third motion cycle for nonpreferred motion was statistically significant (Wilcoxon rank sum test; DB experiment: $p<0.001$; $n=73$; NB experiment: $p<0.05 ; n=33$ ). Thus, the decrease of neuronal responses to nonattended motion in preferred direction clearly contributes to the reduction of directional selectivity, but the main reason for the increasing DI reduction over time is the rising responsiveness of neurons outside the focus of attention to nonpreferred directions of motion.

\section{Discussion}

The aim of the present study was to determine the effects of sustained attention on direction-selective neuronal responses in area MT. We found high and constant neuronal direction selectivity under conditions of attention, whereas in the nonattended case, the DI decreased progressively, reaching significantly 
A

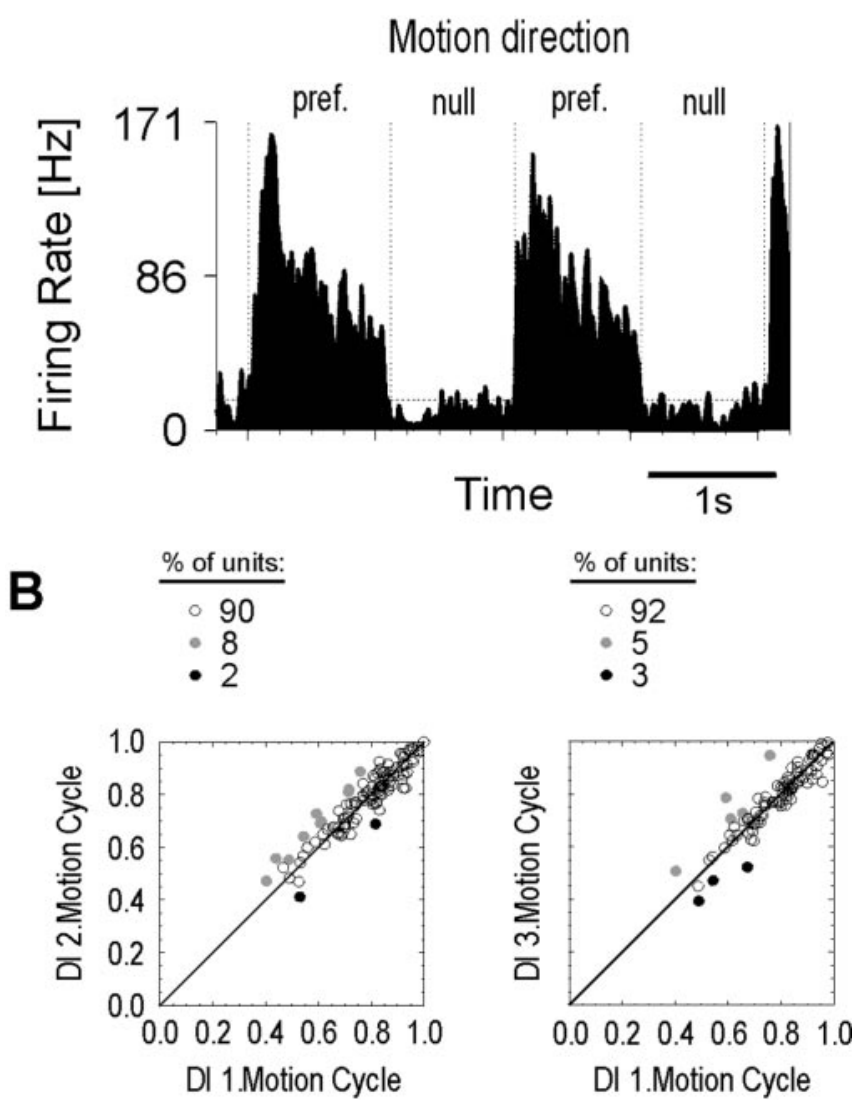

Figure 6. Passive condition. A, PSTH showing the typical response of a neuron to repetitive presentation of backward and forward motion. Conventions are the same as in Figure 2. Note that the general response pattern is preserved over time, leading to essentially constant DIs $(0.8,0.8)$. $B$, The DI of the second and third motion cycles (MCs) versus DI of the first $M C$ in the population of MT neurons. Conventions are the same as in Figure 3. For both periods, the vast majority of neurons showed no statistical change of the DI. Of the remaining cells for which a statistically significant change of the DI was computed, some showed an increase of the DI, and a few suffered from decreased direction selectivity.

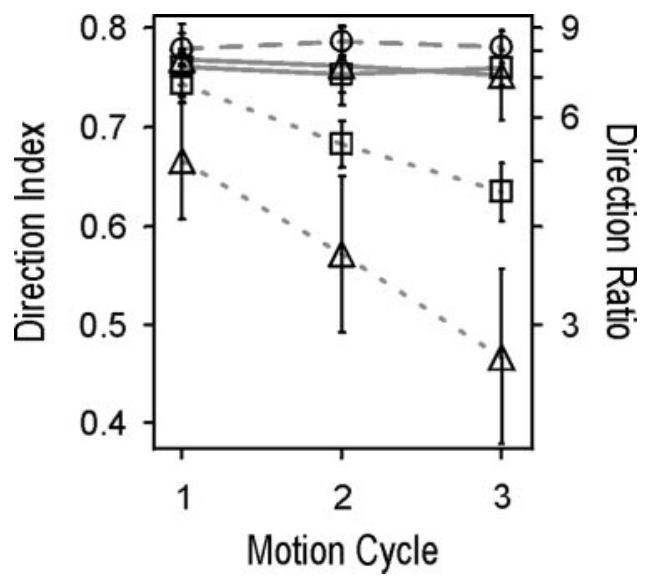

Figure 7. Comparison of average direction selectivity of the populations of neurons recorded under different behavioral conditions. Circles connected with dashed line, Passive condition; squares, DB experiment; triangles, NB experiment; straight lines, attended condition; dotted lines, nonattended condition. For both attended conditions, the plot shows high and constant direction selectivity over time as it was otherwise measured only for the single motion stimulus in the passive condition experiment. In the nonattended conditions, direction selectivity showed a continuous decline over time, which was more pronounced if target and distracter bar were presented in reduced distance. Error bars indicate relative coefficient of variation.
Distant Bar
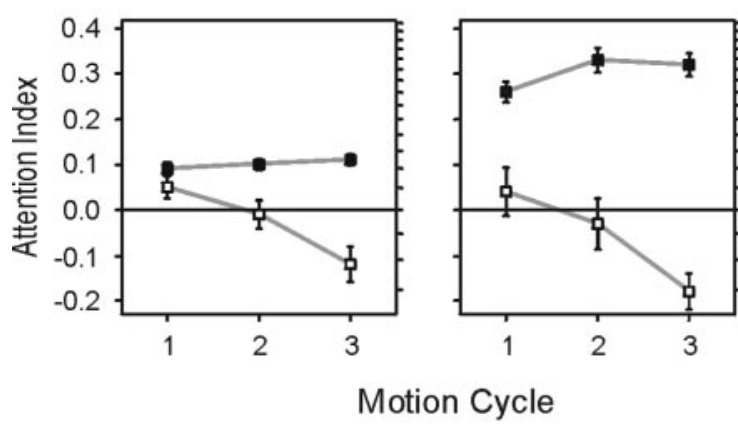

2.4

2.0

1.6

1.4

1.2

1.0

0.9

0.8

0.7

Motion Cycle

Figure 8. Time course of attention index and attention ratio during three consecutive motion cycles for preferred ( $\square$ ) and null ( $\square$ ) motion stimuli. In both experiments, attentional modulation of response rates to preferred motion showed only a moderate increase over time. In contrast, the Al calculated for null motion stimuli exhibits a striking time course, falling from positive values in the first motion cycle to increasingly more negative values in the second and third motion cycles. This indicates that in both experiments, neurons tend to fire more spikes to null motion when the null stimulus is outside the focus of attention. The modulation of responses to null motion is therefore the most important reason for the continuous decline of direction selectivity over time. Error bars indicate SEM.

smaller values than in the attended condition. This timedependent degradation further increased with reduced spatial distance between stimuli and was observed for the majority of cells in the population. The effect was predominantly caused by progressively increasing responses to motion in nonpreferred direction.

For interpretation of the data, the possibility that the strong response modulation for nonattended stimuli has been caused by eye movements that tracked the target bar with low amplitude can be excluded for the following reasons: any such movement would have been restricted to the fixation window, which had a total size of $1.5 \times 1.5^{\circ}$. Such small eye movements have been shown to cause no differences in the average firing rate (Treue and Maunsell, 1999). Moreover, tracking movements would shift the RF in direction of the movement of the target bar. For RF stimulation with a nonattended bar, this results in an enhancement of the effective speed of the distracter. Because we used speeds far below the average optimum in MT (Maunsell and Van Essen, 1983; Lagae et al., 1993), we then expect higher responses to preferred motion and lower responses to null motion for nonattended stimuli. This is opposite to our findings.

High neuronal activity for nonpreferred motion may also be considered to result from contamination of recordings by spikes from unnoticed units preferring the opposite motion direction. For several reasons, this is no plausible explanation. First, observations of attention-dependent modulation of direction selectivity were made in many carefully isolated single units characterized by sharp response profiles. Second, because of the columnar organization of MT (Albright et al., 1984), simultaneous recordings of neurons with different direction preferences are rare. Third, for stimulation in nonpreferred direction, such unnoticed neurons would be expected to respond to their preferred stimulus and hence to be more active in the attended condition. However, the results of the study showed the opposite behavior of neurons, being more active if attention was withdrawn from the stimulus. Therefore, we conclude that the observed change in direction selectivity reflects an attention-dependent property of individual neurons in area MT.

The strong DI modulation raises the question whether attention causes an increase in neuronal selectivity or, alternatively, 
whether selectivity is diminished in the nonattended condition. We conducted a passive condition experiment that was characterized by, first, the absence of a competing motion stimulus and, second, the behavioral insignificance of the moving object. Thus, neuronal resources in MT may be fully attributed to the only motion stimulus in the scene, but a processing benefit from attention mechanisms is unlikely to occur. The mean DI observed in this experiment is therefore expected to indicate the optimal representation of the object without attention. Comparison of the results of this experiment with those from the attended conditions showed a remarkable correspondence of the data, both in terms of absolute strength of direction selectivity and its constancy over time. We conclude that degradation of direction selectivity is a consequence of competition among multiple objects within the same feature domain when one of these objects is the target of attention. With respect to the direction of attentional modulation, this further indicates that, under conditions of competition, attention ensures optimal direction selectivity for neurons representing the behaviorally relevant object.

For this conclusion, it is important to exclude the possibility, that in the passive condition, the monkey paid attention to the single motion stimulus. We think this is improbable because of the following reasons. First, with attention directed to the parafoveally presented bar, a relatively high ratio of eye errors is expected as indicated by the attention experiments. In the passive condition, this was not the case. Rather, for more than half of all recordings, the number of eye errors was zero as expected for pure fixation. Second, if attention would have been directed to the motion stimulus, a similar firing rate as in the attended condition is expected. In contrast, analysis of firing rates (data not shown) found activity patterns clearly below those of the attended conditions for both preferred and null motion in the passive condition, as described previously in the literature (Treue and Martínez Trujillo, 1999). We therefore exclude the possibility that maintenance of direction selectivity in this condition was caused by unexpected attention to the behaviorally irrelevant stimulus.

An important aspect of our study was the finding that the observed DI decrease for nonattended objects had a characteristic time course and became progressively larger. The high and constant DI found in the passive condition shows that this weakened selectivity cannot be interpreted as a general property of neuronal responses independent of attention. A possible explanation for the time course might be an adaptation process, which is prevented for the attended stimulus. For several reasons, such a model is not supported by the data presented here and in the literature. First, epochs of motion not exceeding $1.5 \mathrm{sec}$ between changes of stimulus direction are not expected to induce adaptation (Vautin and Berkley, 1977; Giaschi et al., 1993). Nevertheless, even if some form of short-term adaptation (Priebe and Lisberger, 2002) occurred, the time course of adaptation-induced response changes is expected to repeat within each motion cycle but should not develop continuously over multiple cycles. Second, recent work on adaptation in area MT suggests that in our case, adaptation should rather strengthen direction selectivity instead of weaken it. This follows from the observation that neuronal responses are enhanced when a preferred motion stimulus is preceded by adapting motion in the opposite direction (van Wezel and Britten, 2002; Kohn and Movshon, 2003). Observations for the reverse constellation, nonpreferred preceded by preferred motion, show either reduced activity after long adaptation periods of $20 \mathrm{sec}$ or more (Petersen et al., 1985; Kohn and Movshon, 2003) or unchanged activity after short adaptation periods of $3 \mathrm{sec}$ (van Wezel and Britten, 2002). Thus, none of the reported results supports a decrease of response contrast between preferred and nonpreferred motion stimuli. Therefore, the observed reduction of stimulus selectivity is unlikely to be explained by adaptation of direction selective neurons.

An alternative approach to explain the progressive change of the DI is an attention-dependent modification of the efficacy of neuronal mechanisms promoting direction selectivity. Direction-selective responses are thought to depend on the opposing effects of excitation and inhibition (Mikami et al., 1986a,b). Previous experiments in monkey area MT have found that suppression of responses to null motion predominantly depends on the operation of inhibitory mechanisms (Mikami, 1992). Computer simulations indicated that the strength of these responses, and hence the sharpness of direction selectivity, can be explained by a modulation of the strength of intraareal inhibition (Tanaka and Shinbata, 1994). Therefore, one may hypothesize that attention, beyond its action on excitatory mechanisms, ensures the effectiveness of mutual inhibition between different direction columns. The strength of these mechanisms depends on several factors influencing GABAergic synaptic transmission. Moreover, it was suggested that the efficacy of inhibition is modulated by the strength of synchrony among inhibitory neurons or among their afferent input (Thomson and West, 1993; Gauck and Jaeger, 2000). Attention-dependent changes of neuronal synchronization have been described in different cortical areas (Steinmetz et al., 2000; Fries et al., 2001; Taylor et al., 2003), including area MT (Wegener et al., 2002). Interestingly, during consecutive motion cycles, the strength of synchronization for nonattended stimuli moving in preferred direction was found to decrease over time, whereas with attention, no such attenuation was observed (Wegener, 2004). A reduction of synchronization is expected to result in a progressively reduced efficacy of the inhibitory input to neurons with opposite direction preference, which respond to the same stimulus. Hence, their response to nonpreferred stimuli will continuously increase, thereby causing a degradation of direction selectivity.

Previous investigations of the effect of attention on neuronal tuning in extrastriate areas MT and V4 found that attention modulates responses to preferred and nonpreferred stimuli by the same proportion (McAdams and Maunsell, 1999a; Treue and Martínez Trujillo, 1999). These findings are confirmed by the directly comparable results obtained for the first motion cycle in the DB experiment. With respect to this early part of the trials, there was no significant difference of the AI for preferred and nonpreferred stimuli. However, when attention sustained for periods longer than those investigated in previous work, there was a progressive change of the AIs, making them increasingly different for the two directions of motion. This results in the progressive decrease of direction selectivity for neurons representing the nonattended stimulus.

Attention-dependent modulation of orientation discriminability has recently been described for neurons in area v4 (McAdams and Maunsell, 1999b), although very small in magnitude, and in a psychophysical study (Blanco and Soto, 2002). Evidence for attention-dependent changes on neural selectivity was found in a recent fMRI study on object processing in human lateral occipital complex (Murray and Wojciulik, 2004). Perceptual deficits associated with sustained states of nonattention have been observed in several psychophysical experiments on inattentional blindness (Simons, 2000). These studies described a reduced ability to perceive a stimulus while attention is directed to another object. This failure of perception has been attributed to a degraded neuronal representation of nonattended objects (Rees et al., 1999). Alternatively, it has been argued that it may reflect 
“inattentional amnesia” (Wolfe, 1999). The present results do not exclude the latter possibility but demonstrate a physiological basis for a degraded representation of nonattended stimuli under conditions that are very similar with respect to the requirement for long-lasting allocation of attention.

Together, the present study demonstrates strong effects of sustained attention on the ability of MT neurons to represent the direction of a moving stimulus. Attention serves to enhance cortical processing by maintaining a high level of feature selectivity within groups of neurons representing the attended stimulus, therefore supporting an optimal representation. The finding that neuronal selectivity for simultaneously presented, nonattended stimuli was impaired indicates that perceptual deficits outside the focus of attention may result from reduced cortical processing.

\section{References}

Albright TD, Desimone R, Gross CG (1984) Columnar organization of directionally selective cells in visual area MT of the macaque. J Neurophysiol 51:16-31.

Allik J, Rauk M, Luuk A (1981) Control and sense of eye movement behind closed eyelids. Perception 10:39-51.

Allman J, Miezin F, McGuinness E (1985) Direction- and velocity-specific responses from beyond the classical receptive field in the middle temporal visual area (MT). Perception 14:105-126.

Becklen R, Cervone D (1983) Selective looking and the noticing of unexpected events. Mem Cognit 11:601-608.

Blanco MJ, Soto D (2002) Effects of spatial attention on detection and identification of oriented lines. Acta Psychol (Amst) 109:195-212.

Bour LJ, Van Ginsbergen JAM, Bruijns J, Ottes FP (1984) The double magnetic induction method for measuring eye movement-results in monkey and man. IEEE Trans Biomed Eng BME 31:419-427.

Desimone R, Duncan J (1995) Neural mechanisms of selective visual attention. Annu Rev Neurosci 18:193-222.

Desimone R, Ungerleider LG (1986) Multiple visual areas in the caudal superior temporal sulcus of the macaque. J Comp Neurol 248:164-189.

Fries P, Reynolds JH, Rorie AE, Desimone R (2001) Modulation of oscillatory neuronal synchronization by selective visual attention. Science 291:1560-1563.

Fuchs AF, Robinson DA (1966) A method for measuring horizontal and vertical eye movement chronically in the monkey. J Appl Physiol 21:1068-1070.

Gauck V, Jaeger D (2000) The control of rate and timing of spikes in the deep cerebellar nuclei by inhibition. J Neurosci 20:3006-3016.

Giaschi D, Douglas R, Marlin S, Cynader MS (1993) The time course of direction selective adaptation in simple and complex cells in cat striate cortex. J Neurophysiol 70:2024-2034.

Judge SJ, Richmond BJ, Chu FC (1980) Implantation of magnetic search coils for measurement of eye position: an improved method. Vision Res 20:535-538.

Kohn A, Movshon JA (2003) Neuronal adaptation to visual motion in area MT of the macaque. Neuron 39:681-691.

Lagae L, Raiguel S, Orban GA (1993) Speed and direction selectivity of macaque middle temporal neurons. J Neurophysiol 69:19-39.

Mack A, Rock I (1998) Inattentional blindness. Cambridge, MA: MIT.

Maunsell JH, Gibson JR (1992) Visual response latencies in striate cortex of the macaque monkey. J Neurophysiol 68:1332-1344.

Maunsell JHR, Van Essen DC (1983) Functional properties of neurons in middle temporal visual area of the macaque monkey. I. Selectivity for stimulus direction, speed, and orientation. J Neurosci 3:2563-2586.

May JG, Tsiappoutas KM, Flanagan MB (2003) Disappearance elicited by contrast decrements. Percept Psychophys 65:763-769.

McAdams CJ, Maunsell JHR (1999a) Effects of attention on orientationtuning functions of single neurons in macaque cortical area V4. J Neurosci 19:431-441.

McAdams CJ, Maunsell JHR (1999b) Effects of attention on the reliability of individual neurons in monkey visual cortex. Neuron 23:765-773.
Mikami A (1992) Spatiotemporal characteristics of direction-selective neurons in the middle temporal visual area of the macaque monkeys. Exp Brain Res 90:40-46.

Mikami A, Newsome WT, Wurtz RH (1986a) Motion selectivity in macaque visual cortex. I. Mechanisms of direction and speed selectivity in extrastriate area MT. J Neurophysiol 55:1308-1327.

Mikami A, Newsome WT, Wurtz RH (1986b) Motion selectivity in macaque visual cortex. II. Spatiotemporal range of directional interactions in MT and V1. J Neurophysiol 55:1328-1339.

Most SB, Simons DJ, Scholl BJ, Jimenez R, Clifford E, Chabris CF (2001) How not to be seen: the contribution of similarity and selective ignoring to sustained inattentional blindness. Psychol Sci 12:9-17.

Murray SO, Wojciulik E (2004) Attention increases neural selectivity in the human lateral occipital complex. Nat Neurosci 7:70-74.

Neisser U (1967) Cognitive psychology. New York: Appleton-Century-Crofts.

Neisser U, Becklen R (1975) Selective looking: attending to visually specified events. Cognit Psychol 7:480-494.

Petersen SE, Baker JF, Allman JM (1985) Direction-specific adaptation in area MT of the owl monkey. Brain Res 346:146-150.

Priebe NJ, Lisberger SG (2002) Constraints on the source of short-term motion adaptation in macaque area MT. II. Tuning of neural circuit mechanisms. J Neurophysiol 88:370-382.

Rees G, Russell C, Frith CD, Driver J (1999) Inattentional blindness versus inattentional amnesia for fixated but ignored words. Science 24:2504-2507.

Simons DJ (2000) Attentional capture and inattentional blindness. Trends Cogn Sci 4:147-155.

Simons DJ, Chabris CF (1999) Gorillas in our midst: sustained inattentional blindness for dynamic events. Perception 28:1059-1074.

Steinmetz PN, Roy A, Fitzgerald PJ, Hsiao SS, Johnson KO, Niebur E (2000) Attention modulates synchronized neuronal firing in primate somatosensory cortex. Nature 404:187-190.

Stoffregen TA, Baldwin CA, Flynn SB (1993) Noticing of unexpected events by adults with and without mental retardation. Am J Ment Retard 98:273-284.

Tanaka S, Shinbata H (1994) Mathematical model for self-organization of direction columns in the primate middle temporal area. Biol Cybern 70:227-234.

Taylor K, Mandon S, Freiwald W, Kreiter AK (2003) Attention strongly increases neuronal synchrony in V4 in a shape tracking task. Soc Neurosci Abstr 29:385.11.

Thomson AM, West DC (1993) Fluctuations in pyramid-pyramid excitatory postsynaptic potentials modified by presynaptic firing pattern and postsynaptic membrane potential using paired intracellular recordings in rat neocortex. Neuroscience 54:329-346.

Treue S, Martínez Trujillo JC (1999) Feature-based attention influences motion processing gain in macaque visual cortex. Nature 399:575-579.

Treue S, Maunsell JHR (1996) Attentional modulation of visual motion processing in cortical areas MT and MST. Nature 382:539-541.

Treue S, Maunsell JHR (1999) Effects of attention on the processing of motion in macaque visual cortical areas MT and MST. J Neurosci 19:7591-7602.

van Wezel RJA, Britten KH (2002) Motion adaptation in area MT. J Neurophysiol 88:3469-3476.

Vautin RG, Berkley MA (1977) Responses of single cells in cat visual cortex to prolonged stimulus movement: neural correlates of visual aftereffects. J Neurophysiol 40:1051-1065.

Wegener D (2004) The influence of selective visual attention on neuronal synchronization and temporal activity patterns in the parietal cortex of the macaque. PhD thesis, Wissenschaftlicher Verlag Berlin.

Wegener D, Freiwald WA, Kreiter AK (2002) Selective visual attention affects synchronisation and oscillatory patterns in macaque area MT. Soc Neurosci Abstr 28:559.8.

Wegener D, Freiwald WA, Kreiter AK (2003) Attention and stimulus specificity in macaque area MT. Soc Neurosci Abstr 29:385.9.

Wolfe JM (1999) Inattentional amnesia. In: Fleeting memories (Coltheart V, ed), pp 1-12. Cambridge, MA: MIT. 